\title{
A Weakly Robust PTAS for Minimum Clique Partition in Unit Disk Graphs
}

\author{
Imran A. Pirwani*
}

\author{
Mohammad R. Salavatipour ${ }^{\dagger}$
}

\begin{abstract}
We consider the problem of partitioning the set of vertices of a given unit disk graph (UDG) into a minimum number of cliques. The problem is NP-hard and various constant factor approximations are known, with the current best ratio of 3 . Our main result is a weakly robust polynomial time approximation scheme (PTAS) for UDGs expressed with edge-lengths, it either (i) computes a clique partition or (ii) gives a certificate that the graph is not a UDG; for the case (i) that it computes a clique partition, we show that it is guaranteed to be within $(1+\varepsilon)$ ratio of the optimum if the input is UDG; however if the input is not a UDG it either computes a clique partition as in case (i) with no guarantee on the quality of the clique partition or detects that it is not a UDG. Noting that recognition of UDG's is NP-hard even if we are given edge lengths, our PTAS is a weakly-robust algorithm. Our algorithm can be transformed into an $O\left(\frac{\log ^{*} n}{\varepsilon^{O(1)}}\right)$ time distributed PTAS.

We consider a weighted version of the clique partition problem on vertex weighted UDGs that generalizes the problem. We note some key distinctions with the unweighted version, where ideas useful in obtaining a PTAS breakdown. Yet, surprisingly, it admits a $(2+\varepsilon)$-approximation algorithm for the weighted case where the graph is expressed, say, as an adjacency matrix. This improves on the best known 8-approximation for the unweighted case for UDGs expressed in standard form.
\end{abstract}

Keywords: Computational Geometry, Approximation Algorithms.

\section{Introduction}

A standard network model for homogeneous networks is the unit disk graph (UDG). A graph $G=(V, E)$ is a UDG if there is a mapping $f: V \mapsto \mathbb{R}^{2}$ such that $\|f(u)-f(v)\|_{2} \leq 1 \Leftrightarrow\{u, v\} \in E$; $f(u)^{1}$ models the position of the node $u$ while the unit disk centered at $f(u)$ models the range of radio communication. Two nodes $u$ and $v$ are said to be able to directly communicate if they lie in the unit disks placed at each others' centers. There is a vast collection of literature on algorithmic problems studied on UDGs. See the survey [2].

Clustering of a set of points is an important subroutine in many algorithmic and practical applications and there are many kinds of clusterings depending upon the application. A typical objective in clustering is to minimize the number of "groups" such that each "group" (cluster)

\footnotetext{
*Department of Computing Science, University of Alberta, Edmonton, Alberta T6G 2E8, Canada. Email: pirwani@cs.ualberta.ca. Supported by Alberta Ingenuity.

${ }^{\dagger}$ Department of Computing Science, University of Alberta, Edmonton, Alberta T6G 2E8, Canada. Email: mreza@cs.ualberta.ca. Supported by NSERC and Alberta Ingenuity.

${ }^{1} f($.$) is called a realization of G$. Note that $G$ may not come with a realization.
} 
satisfies a set of criteria. Mutual proximity of points in a cluster is one such criterion, while points in a cluster forming a clique in the underlying network is an extreme form of mutual proximity. We study an optimization problem related to clustering, called the minimum clique partition problem on this UDGs.

Minimum clique partition on unit disk graphs (MCP): Given a unit disk graph, $G=$ $(V, E)$, partition $V$ into a smallest number of cliques.

Despite being theoretically interesting, MCP has been useful for other problems. For example, 17. shows how to use a small-sized clique partition of a UDG to construct a large collection of disjoint (almost) dominating sets. They [18 also show how to obtain a good quality realization of UDGs, and an important ingredient in their technique was to construct a small-sized clique partition of the graph. It is shown [12] how to use a small-sized clique partition to obtain sparse spanners with bounded dilation, which also permit guaranteed geographic routing on a related class of graphs. 114 employ MCP to obtain an $O\left(\log ^{*} n\right)$ time distributed algorithm which is an $O(\log n)$-approximation for the facility location problem on UDGs without geometry; they also give an $O(1)$ time distributed $O(1)$-approximation to the facility location problem on UDGs with geometry also using MCP. Recently, [15] shows how to obtain a first $O(1)$ approximation to the domatic partition problem on UDGs using MCP.

On general graphs, the clique-partition problem is equivalent to the minimum graph coloring on the complement graph which is not approximable within $n^{1-\varepsilon}$, for any $\varepsilon>0$, unless $\mathrm{P}=\mathrm{NP}$ 22. MCP has been studied for special graph classes. It is shown to be MaxSNP-hard for cubic graphs and NP-complete for planar cubic graphs [5]; they also give a 5/4-approximation algorithm for graphs with maximum degree at most 3. MCP is NP-hard for a subclass of UDGs, called unit coin graphs, where the interiors of the associated disks are pairwise disjoint [6]. Good approximations, however, are possible on UDGs. The best known approximation is due to [6] who give a 3-approximation via a partitioning the vertices into co-comparability graphs, and solving the problem exactly on them. They give a 2-approximation algorithm for coin graphs. MCP has also been studied on UDGs expressed in standard form. For UDGs expressed in general form [18 give an 8-approximation algorithm.

\section{Our Results and Techniques:}

In this paper we present a weakly-robust2 PTAS for MCP on a given UDG. For ease of exposition, first we prove this (in Section 2.1) when the UDG is given with a realization, $f($.$) . The holy-grail is$ a PTAS when the UDG is expressed in standard form, say, as an adjacency matrix. However, falling short of proving this, we show (in Section 2) how to get a PTAS when the input UDG is expressed in standard form along with associated edge-lengths corresponding to some (unknown) realization. The algorithm is weakly-robust in the sense that it either (i) computes a clique partition of the input graph or (ii) gives a certificate that the input graph is not a UDG. If the input is indeed a UDG then the algorithm returns a clique partition (case (i)) which is a $(1+\varepsilon$ )-approximation (for a given $\epsilon>0$ ). However, if the input is not a UDG, the algorithm either computes a clique partition but with no guarantee on the quality of the solution or returns that it is not a UDG. Therefore, this

\footnotetext{
${ }^{2} \mathrm{An}$ algorithm is called robust if it either computes an answer or declares that the input is not from the restricted domain; if the algorithm computes an answer then it is correct 20. We call our algorithm weakly-robust in that it always computes a clique partition or declares that the input is not from the restricted domain (i.e. not a UDG); if the input happens to be a UDG then the answer is a $(1+\epsilon)$-approximate clique partition. Otherwise, it still returns a clique partition but there is no guarantee on the quality of the clique partition.
} 
algorithm should be seen as a weakly-robust PTAS. The generation of a polynomial-sized certificate which proves why the input graph is not a UDG should be seen in the context of the negative result of [1] which says that even if edge lengths are given, UDG recognition is NP-hard. We show (in Section (4) how this algorithm can be modified to run in $O\left(\frac{\log ^{*} n}{\varepsilon^{O(1)}}\right)$ distributed rounds.

In Section 3 we explore a weighted version of MCP where we are given a vertex weighted UDG. In this formulation, the weight of a clique is the weight of a heaviest vertex in it, and the weight of a clique partition is the sum of the weights of the cliques in it. We note some key distinctions between the weighted and the unweighted versions of the problem and show that the ideas that help in obtaining a PTAS do not help in the weighted case. Yet, surprisingly, we show that the problem admits a $(2+\varepsilon)$-approximation algorithm for the weighted case using only adjacency. This result should be contrasted with the unweighted case where it is not clear as to how to remove the dependence on the use of edge-lengths, which was crucially exploited in deriving a PTAS.

We use OPT to denote an optimum clique partition and opt to denote the size (or, in Section 3 , weight) of an optimum clique partition. We also use $n$ and $m$ to denote the number of points (i.e. nodes of $G=(V, E))$ and the number of edges, respectively.

\section{A Weakly-Robust PTAS for UDG Expressed with Edge-lengths}

For simplicity, we first describe an algorithm when the input is given with a geometric realization.

\subsection{A PTAS for UDGs With a Geometric Realization}

We assume the input UDG is expressed with geometry of its points. Using a randomly shifted grid whose cell size is $k \times k$ (for $k=k(\varepsilon)$ ) we partition the plane. Since the diameter of the convex hull of each clique is at most 1 , for large values of $k$, a fixed clique is cut by this grid (and therefore belongs to at most four cells) with probability at most $\frac{2}{k}$. Therefore, if we could efficiently compute an optimal clique partition in each $k \times k$ cell, then taking the union of these cliques yields a solution whose expected size at most $(1+\varepsilon)$ opt. We can easily repeat this process $O(\log n)$ times to obtain a solution with size at most $(1+\varepsilon)$ opt w.h.p. We call the algorithm MinCP1, formalized below.

Theorem 1. Algorithm MinCP1 (given below) returns, in poly-time, a clique partition of size at most $(1+\varepsilon)$ opt w.h.p.

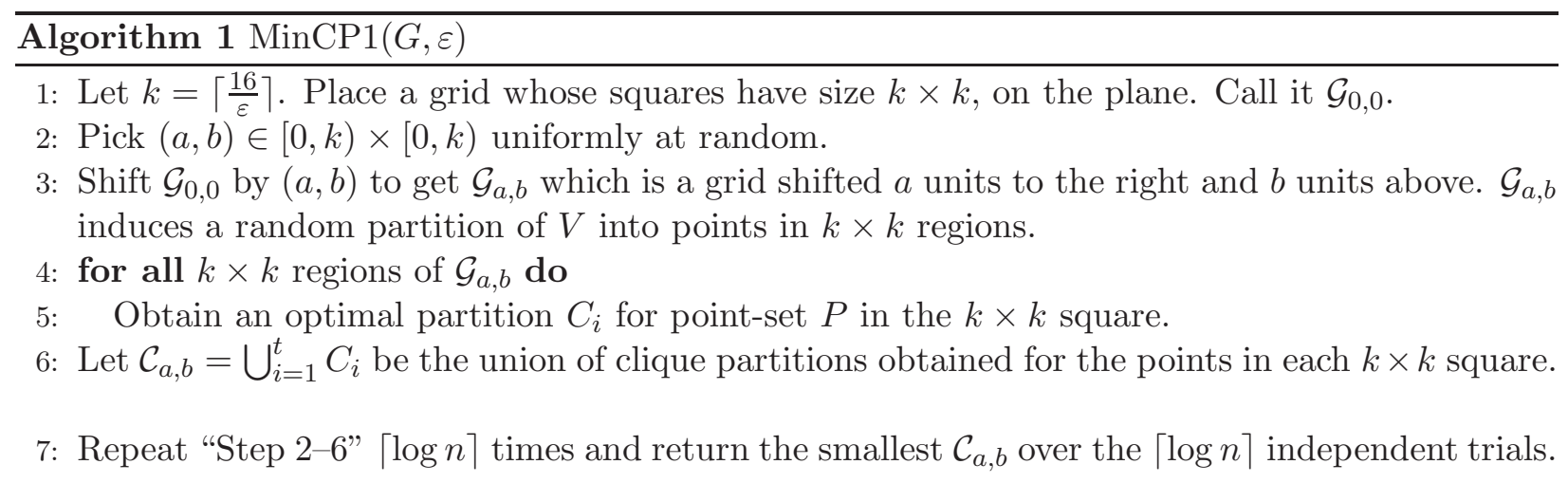

We begin with a simple observation. 
Observation 2. The diameter of the convex hull of every clique is at most 1.

In the next subsection we argue how to perform "Step 5" of the algorithm MinCP1 efficiently. Assuming this, we prove Theorem 1. For a random shift $\mathcal{G}_{a, b}$ and a clique $C$, we say that $\mathcal{G}_{a, b}$ "cuts" $C$ if some line of $\mathcal{G}_{a, b}$ crosses an edge of $C$. It is easy to see that:

$$
\operatorname{Pr}\left[\begin{array}{c}
C \text { is cut } \\
\text { by } \mathcal{G}_{a, b}
\end{array}\right] \leq \operatorname{Pr}\left[\begin{array}{c}
\text { a vertical or horizontal line of } \mathcal{G}_{a, b} \\
\text { crosses an edge of } C
\end{array}\right] \leq \frac{2}{k}
$$

Thus, the expected number of cliques in an optimal partition that are "cut" by $\mathcal{G}_{a, b}$ is at most $\frac{2}{k}$. opt. So, by Markov's inequality, with probability at least $1 / 2$ there are no more than $\frac{4}{k} \cdot$ opt cliques cut by $\mathcal{G}_{a, b}$. Therefore, if we compute an optimal solution for each of the $k \times k$ grid cells and take the union of them, with probability at least $1 / 2$ we get an excess of at most $4 \times \frac{4}{k}$. opt cliques with respect to optimum since each clique that is "cut" by the grid can be counted up to four times. If we repeat this process for $\lceil\log n\rceil$ independent random trials, we get that with probability at least $1-\frac{1}{n}$ the size of the solution we obtain is at most opt $+\frac{16}{k}$. opt $\leq(1+\varepsilon)$. opt.

\subsubsection{Optimal Clique Partition of a UDG in a $k \times k$ Square}

Unlike optimization problems such as maximum (weighted) independent set and minimum dominating set, where one can "guess" only a small-sized subset of points to obtain an optimal solution, the combinatorial complexity of any single clique in an optimal solution can be high. Therefore, it is unclear as to how to "guess" even few cliques, each of which may be large. A result of Capoyleas et al. 4 comes to our aid; a version of their result says that there exists an optimal clique partition where the convex hulls of the cliques are pair-wise non-overlapping. This phenomenon of separability of an optimal partition, coupled with the fact that the size of an optimal partition in a small region is small, allows us to circumvent the above difficulty. The following simple lemma bounds the size of an optimal solution of an instance of bounded diameter.

Lemma 3. Any set of points $P$ in a $k \times k$ square has a clique partition of size $O\left(k^{2}\right)$.

Proof. Place a grid whose cells have size $1 / 2 \times 1 / 2$. This grid induces a vertex partition where each block in the partition consists of the points that share a common grid cell (and therefore form a clique).

We state a variant of a result by Capoyleas et al. 4 according to which there exists an optimal clique partition where the convex hulls of the cliques are non-overlapping, that is, for any pair of cliques in an optimal partition, there is a straight line which separates them 3

Theorem 4 (4]). For a clique partition in which the convex hulls of the cliques are pairwise nonoverlapping, there is a straight line $l_{i j}$ that separates a pair of cliques $C_{i}, C_{j}$ such that all vertices of $C_{i}$ are on one side of $l_{i j}$, and all the vertices of $C_{j}$ are on the other side of $l_{i j}$. (see Figure 11). Furthermore, this partition can be computed in poly-time.

The general structure of the algorithm for computing optimal solution of a $k \times k$ cell is as follows. In order to reduce the search space for separator lines, one can find a characterization of the separator lines with some extra properties. Let $C_{i}, C_{j}$ be a pair of cliques each having at least

\footnotetext{
${ }^{3}$ We gave a proof of this theorem [19] before it was brought to our attention that Capoyleas, Rote, and Woeginger 4 proved this much earlier in a different context.
} 


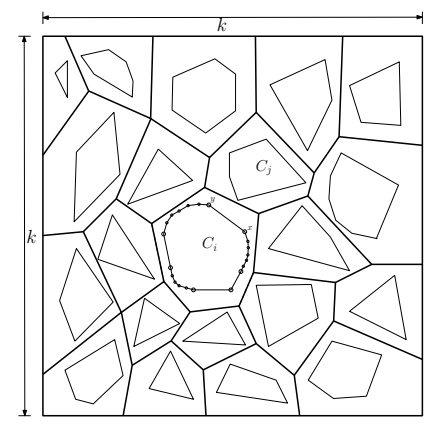

(a)

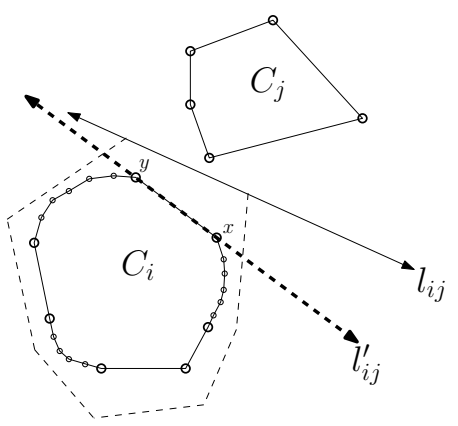

(b)

Figure 1: (a) An optimal clique partition of UDG points in a bounded region; each light convex shape corresponds to a clique in the clique partition. The heavy line-segments represent segments of the corresponding separators. (b) A close-up view of $C_{i}$ and $C_{j}$. A separator line, $l_{i j}$ is shown which separates $C_{i}$ and $C_{j}$, corresponding to the segment in (a). Note that $l_{i j}^{\prime}$ is also a separator for $C_{i}$ and $C_{j}$ and $l_{i j}^{\prime}$ is passing through points $x$ and $y$ in $C_{i}$.

two points. Let $L_{i j}$ be the (infinite) set of distinct separator lines. Since $C_{i}$ and $C_{j}$ are convex, there exists at least one line in $L_{i j}$ that goes through two points of $C_{i}$ (or $C_{j}$ ) (see Figure 1(b)). Therefore, given two cliques $C_{i}$ and $C_{j}$ in a clique partition (with pairwise non-overlapping parts) there is a separator line $l_{i j}$ that goes through two vertices of one of them, say $u, v \in C_{i}$ such that all the vertices of $C_{j}$ are on one side of this line and all the vertices of $C_{i}$ are on the other side or on the line. Since there are $O\left(k^{2}\right)$ cliques in an optimal partition of $k \times k$ cell, there are $O\left(k^{4}\right)$ pairs of cliques in the partition and their convex hulls are pairwise non-overlapping. In fact, a more careful analysis shows that the dual graph of the regions is planar (see Figure 1(a)); thus there are $O\left(k^{2}\right)$ distinct straight lines, each of which separate a pair of cliques in our optimal solution. For every clique $C_{i}$, the separator lines $l_{i j}$ (for all values of $j$ ) define a convex region that contains clique $C_{i}$. So once we guess this set of $O\left(k^{2}\right)$ lines, these convex regions define the cliques. We will try all possible (non-equivalent) sets of $O\left(k^{2}\right)$ separator lines and check if each of the convex regions indeed defines a clique and if we obtain a clique partition. This can be performed in $O\left(n^{k^{2}}\right)$ time (see [4] for more details).

\subsection{A PTAS for UDGs With Edge-Lengths Only}

We weaken our assumption on having access to geometry; we assume only edge-lengths are known with respect to a feasible (unknown) realization of the UDG. We prove that,

Theorem 5. Given a graph $G$ with associated (rational) edge-lengths and $\varepsilon>0$, there is a polynomial time algorithm which either computes a clique partition of $G$ or gives a certificate that $G$ is not a UDG. If $G$ is a $U D G$, the size of the clique partition computed is a $(1+\varepsilon)$-approximation of the optimum clique partition (but there is no guarantee on the size of the clique partition if the input graph is not $U D G$ ).

The high level idea of the algorithm is as follows. As in the geometric case, we first decompose the graph into bounded diameter regions and show that if we can compute the optimum clique partition of each region then the union of these clique partitions is within $(1+\varepsilon)$ fraction of the optimum. There are two main difficulties here for which we need new ideas. The first major 
difference is that, we cannot use the random shift argument as in the geometric case. To overcome this, we use a ball growing technique that yields bounded diameter regions. This is inspired by 13. who give local PTAS for weighted independent set, and minimum dominating set for UDGs without geometry. The second major difference is that, even if we have the set of points belonging to a bounded region (a ball) it is unclear as to how to use the separation theorem to obtain an optimal solution for this instance. Note that we are not guaranteed to have a UDG as input. We show that we can either compute a clique partition for each subgraph induced by a ball, or give a certificate that the subgraph is not UDG. If it is a UDG, then our clique partition is optimal but if it is not a UDG there is no guarantee on its size.

Let $B_{r}(v)=\{u: d(u, v) \leq r\}$, where by $d(u, v)$ we mean the number of edges on a shortest path from $u$ to $v$. So, $B_{r}(v)$ can be computed using a breadth-first search (BFS) tree rooted at $v$. We describe our decomposition algorithm which partitions the graph into bounded diameter subgraphs in Algorithm 2. We will describe a procedure, called OPT-CP which, given a graph induced by the vertices of $B_{r}(v)$ and a parameter $\ell=\operatorname{poly}(r)$, runs in time $\left|B_{r}(v)\right|^{O\left(\ell^{2}\right)} \leq n^{O\left(\ell^{2}\right)}$ and either produces a certificate that $B_{r}(v)$ is not a UDG or computes a clique partition of $B_{r}(v)$; this clique partition is optimum if $B_{r}(v)$ is a UDG. We only call this procedure for "small" values of $r$.

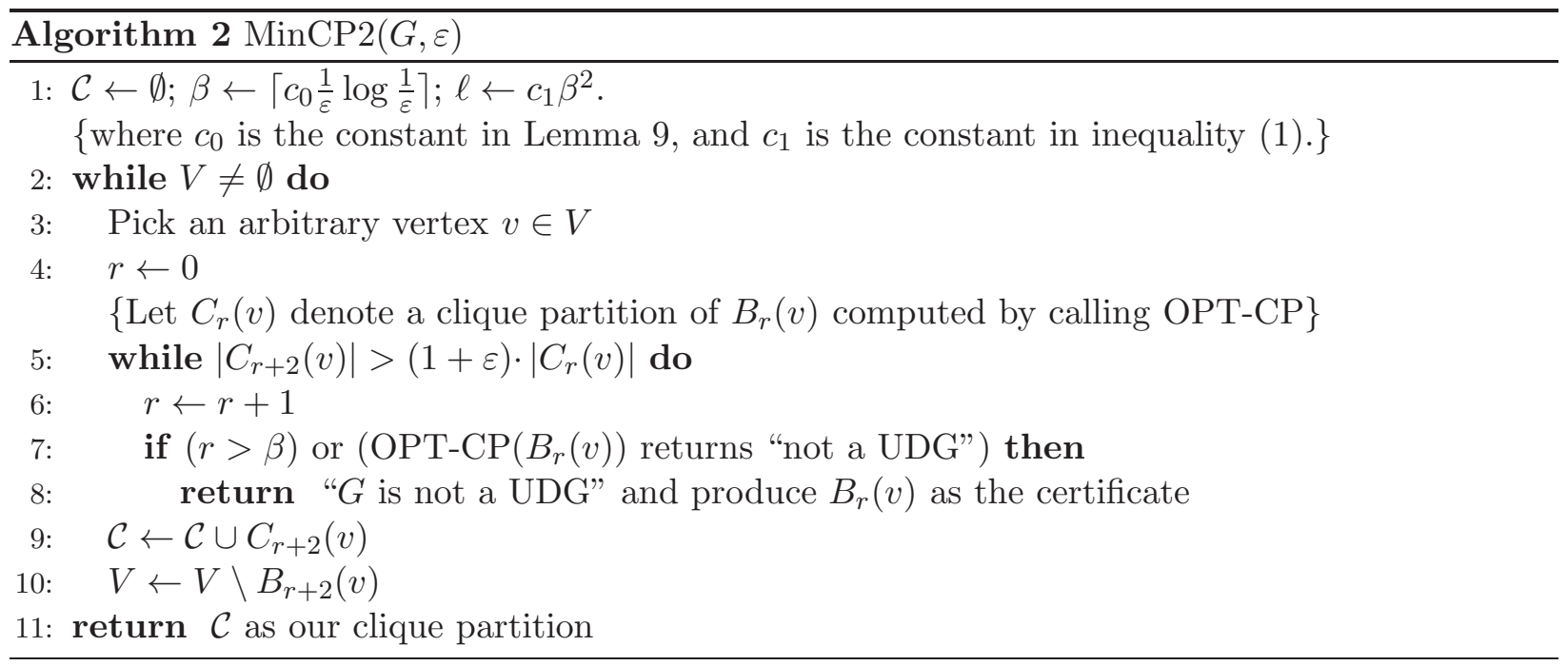

Clearly, if the algorithm returns $\mathcal{C}$ on "Step 11", it is a clique partition. Let us assume that each ball $B_{r}(v)$ we consider induces a UDG and that the procedure OPT-CP returns an optimal clique partition $C_{r}(v)$ for ball $B_{r}(v)$. We show that in this case $|\mathcal{C}| \leq(1+\varepsilon)$ opt. We also show that for any iteration of the outer "while-loop", "Step 5" of MinCP2 is executed in time polynomial in $n$, by using edge-lengths instead of Euclidean coordinates.

For an iteration $i$ of the outer loop, let $v_{i}$ be the vertex chosen in "Step 3" and let $r_{i}^{*}$ be the value of $r$ for which the "while-loop" on "Step 5" terminates, that is, $\left|C_{r_{i}^{*}+2}\left(v_{i}\right)\right| \leq(1+\varepsilon) \cdot\left|C_{r_{i}^{*}}\left(v_{i}\right)\right|$. Let $k$ be the maximum number of iterations of the outer loop. The following lemmas show that two distinct balls grown around vertices are far from each other, that the union of the optimal solutions to the balls form a lower-bound on the cost of the entire instance, and that the cost of $\mathcal{C}$ and opt is within a factor $(1+\varepsilon)$ of opt.

Lemma 6. For every $i \neq j$, every pair $v \in B_{r_{i}^{*}}\left(v_{i}\right)$ and $u \in B_{r_{j}^{*}}\left(v_{j}\right)$ are non-adjacent. 
Proof. Without loss of generality, let $i<j$. Therefore, every vertex in $B_{r_{j}^{*}}\left(v_{j}\right)$ is at a level larger than $r_{i}^{*}+2$ of the BFS tree rooted at $v_{i}$, otherwise it would have been part of the ball $B_{r_{i}^{*}+2}\left(v_{i}\right)$ thus removed from $V$. Note that in a BFS tree rooted at $v_{i}$, there cannot be an edge between a level $r$ and $r^{\prime}$ with $r^{\prime} \geq r+2$. Thus there cannot be an edge between a node in $v \in B_{r_{i}^{*}}\left(v_{i}\right)$, which has level at most $r_{i}^{*}$ and a node $u \in B_{r_{j}^{*}}\left(v_{j}\right)$, which would been at a level at least $r_{i}^{*}+3$ in the BFS tree rooted at $v_{i}$.

Next, we derive a lower-bound on opt.

Lemma 7. opt $\geq \sum_{i=1}^{k}\left|C_{r_{i}^{*}}\left(v_{i}\right)\right|$

Proof. Note that $B_{r_{i}^{*}}\left(v_{i}\right)$ is obtained by constructing a BFS tree rooted at vertex $v_{i}$ up to some depth $r_{i}^{*}$. According to Lemma 6, there is no edge between any two nodes $v \in B_{r_{i}^{*}}\left(v_{i}\right)$ and $u \in B_{r_{j}^{*}}\left(v_{j}\right)$. So, no single clique in an optimum solution can contain vertices from distinct $B_{r_{i}^{*}}\left(v_{i}\right)$ and $B_{r_{i}^{*}}\left(v_{j}\right)$. Consider the subset of cliques in an optimal clique partition of $G$ that intersect $B_{r_{i}^{*}}\left(v_{i}\right)$ and call this subset $\mathrm{OPT}_{i}$. The argument above shows that $\mathrm{OPT}_{i}$ is disjoint from $\mathrm{OPT}_{j}$. Also, each $\mathrm{OPT}_{i}$ contains all the vertices in $B_{r_{i}^{*}}\left(v_{i}\right)$. Since $C_{r_{i}^{*}}\left(v_{i}\right)$ is an optimal clique partition for $B_{r_{i}^{*}}\left(v_{i}\right),\left|\mathrm{OPT}_{i}\right| \geq\left|C_{r_{i}^{*}}\left(v_{i}\right)\right|$. The lemma immediately follows by observing that $\mathrm{OPT}_{i}$ and $\mathrm{OPT}_{j}$ are disjoint.

The next lemma relates the cost of our solution to opt.

Lemma 8. If $\left|C_{r_{i}^{*}+2}\left(v_{i}\right)\right| \leq(1+\varepsilon) \cdot\left|C_{r_{i}^{*}}\left(v_{i}\right)\right|$, then $\left|\bigcup_{i=1}^{k} C_{r_{i}^{*}+2}\left(v_{i}\right)\right| \leq(1+\varepsilon) \cdot$ opt

Proof. $\left|\bigcup_{i=1}^{k} C_{r_{i}^{*}+2}\left(v_{i}\right)\right|=\sum_{i=1}^{k}\left|C_{r_{i}^{*}+2}\left(v_{i}\right)\right| \leq(1+\varepsilon) \cdot \sum_{i=1}^{k}\left|C_{r_{i}^{*}}\left(v_{i}\right)\right| \leq(1+\varepsilon) \cdot$ opt

Finally, we show that the inner "while-loop" terminates in $\tilde{O}\left(\frac{1}{\varepsilon}\right)$, so $r_{i}^{*} \in \tilde{O}\left(\frac{1}{\varepsilon}\right)$. Obviously, the "while-loop" on "Step 5" terminates eventually, so $r_{i}^{*}$ exists. By definition of $r_{i}^{*}$, for all smaller values of $r<r_{i}^{*}:\left|C_{r}\left(v_{i}\right)\right|>(1+\varepsilon) \cdot\left|C_{r-2}\left(v_{i}\right)\right|$. Since diameter of $B_{r}\left(v_{i}\right)$ is $O(r)$, if $B_{r}(v)$ is a UDG, there is a realization of it in which all the points fit into a $r \times r$ grid. Thus, $\left|C_{r}\left(v_{i}\right)\right| \in O\left(r^{2}\right)$. So for some $\alpha \in O(1)$ :

$$
\alpha \cdot r^{2}>\left|C_{r}\left(v_{i}\right)\right|>(1+\varepsilon) \cdot\left|C_{r-2}\left(v_{i}\right)\right|>\ldots>(1+\varepsilon)^{\frac{r}{2}} \cdot\left|C_{0}\left(v_{i}\right)\right|=O\left((\sqrt{1+\varepsilon})^{r}\right),
$$

when $r$ is even (for odd values of $r$ we obtain $\left|C_{r}\left(v_{i}\right)\right|>(1+\varepsilon)^{\frac{r-1}{2}} \cdot\left|C_{1}\left(v_{i}\right)\right| \geq O\left((\sqrt{1+\varepsilon})^{r-1}\right)$. Therefore we have:

Lemma 9. There is a constant $c_{0}>0$ such that for each $i: r_{i}^{*} \leq c_{0} / \varepsilon \cdot \log 1 / \varepsilon$.

In the next subsection, we show that the algorithm OPT-CP, given $B_{r}(v)$ and an upper bound $\ell$ on $\left|C_{r}(v)\right|$, either computes a clique partition or declares that the graph is not UDG; the size of the partition is optimal if $B_{r}(v)$ is a UDG. The algorithm runs in time $n^{O\left(\ell^{2}\right)}$. By the above arguments, if $B_{r}(v)$ is a UDG then, there is a constant $c_{1}>0$ such that:

$$
\left|C_{r}(v)\right|=O\left(r_{i}^{* 2}\right) \leq c_{1} \cdot \frac{c_{0}^{2}}{\varepsilon^{2}} \log ^{2} \frac{1}{\varepsilon} .
$$


We can set $\ell=\left\lceil c_{1} \frac{c_{0}^{2}}{\varepsilon^{2}} \log ^{2} \frac{1}{\varepsilon}\right\rceil$ for any invocation of OPT-CP as an upper bound, where $c_{1}$ is the constant in $O\left(r_{i}^{* 2}\right)$. So, the running time of the algorithm is $n^{\tilde{O}\left(1 / \varepsilon^{4}\right)}$.

\subsection{An Optimal Clique Partition for $B_{r}(v)$}

Here we present the algorithm OPT-CP that given $B_{r}(v)$ (henceforth referred to as $G^{\prime}$ ) and an upper bound $\ell$ on the size of an optimal solution for $G^{\prime}$, either computes a clique partition of it or detects that it is not a UDG; if $G^{\prime}$ is a UDG then the partition is optimal. The algorithm runs in time $n^{O\left(\ell^{2}\right)}$. Since, by Lemma 9, $\ell$ is a constant in each call to this algorithm, the running time of OPT-CP is polynomial in $n$. Our algorithm is based on the separation theorem [4]. Even though we do not have a realization of the nodes on the plane, assuming that $G^{\prime}$ is a UDG, we show how to apply the separation theorem [4] as in the geometric setting. We use node/point to refer to a vertex of $G^{\prime}$ and/or its corresponding point on the plane for some realization of $G^{\prime}$. We will use the following technical lemma.

Lemma 10. Suppose we have four mutually adjacent nodes $p, a, b, r$ and their pairwise distances with respect to some realization on the Euclidean plane. Then there is a poly-time procedure that can decide if $p$ and $r$ are on the same side of the line that goes through $a$ and $b$ or are on different sides.

Proof. First, we describe how to detect if the quadrilateral on these four points is convex or concave. If the quadrilateral is concave, then one of the points will be inside the triangle formed by the other three. There are three possible cases: $r$ is inside, $p$ is inside, or one of $a$ or $b$ is inside (see Figure 2(c)-(e)). There are four triangles each of which is over three of these four points. The quadrilateral is concave if the sum of the areas of three of these triangles is equal to the area of the fourth triangle. Equivalently, it is convex if sum of areas of two of the triangles is equal to the sum of areas of the other two. Given a triangle with edge lengths $x, y, z$, using Heron's formula, the area of the triangle is equal to $\sqrt{\left.2\left(x^{2} y^{2}+y^{2} z^{2}+z^{2} x^{2}\right)-\left(x^{4}+y^{4}+z^{4}\right)\right) / 4}$. So the area of a triangle is of the form $\sqrt{A}$ where $A$ is a polynomial in terms of lengths of the edges of the triangle. Suppose that the areas of the four triangles over these four points are $\sqrt{A_{1}}, \sqrt{A_{2}}, \sqrt{A_{3}}$, and $\sqrt{A_{4}}$. We need to check if the sum of two is equal to the sum of the other two and we would like to do this without computing the square roots of numbers. For instance, suppose we want to verify $\sqrt{A_{1}}+\sqrt{A_{2}}=\sqrt{A_{3}}+\sqrt{A_{4}}$. For this to hold, we must have $A_{1}+A_{2}+2 \sqrt{A_{1} A_{2}}=A_{3}+A_{4}+2 \sqrt{A_{3} A_{4}}$. Verifying this is equivalent to verifying $D+\sqrt{A_{1} A_{2}}=\sqrt{A_{3} A_{4}}$ where $D=\frac{1}{2}\left(A_{1}+A_{2}-A_{3}-A_{4}\right)$. Taking the square of both sides, we need to have $D^{2}+A_{1} A_{2}+2 D \sqrt{A_{1} A_{2}}=A_{3} A_{4}$, which is the same as $\frac{1}{4}\left(A_{3} A_{4}-D^{2}-A_{1} A_{2}\right)^{2}=A_{1} A_{2} D^{2}$. Thus by comparing two polynomials of edge-lengths (and without computing square roots) we can check if the quadrilateral is convex or concave.

Suppose the quadrilateral is convex. If $r$ and $p$ are on two opposite corners (see Figure 2(a)), then $r$ and $p$ are on different sides. In this case $|r p|+|a b|>|r a|+|b p|$ and $|r p|+|a b|>|r b|+|a p|$. If $r p$ is one of its sides (see Figure 2(b)), then $|r p|+|a b|$ is not the largest of the above three pairs of sums.

Now suppose that the quadrilateral is concave. The only case in which $r$ and $p$ are on two sides of line $a b$ is when one of $a$ or $b$ is inside the triangle obtained by the other three (see Figure $2(\mathrm{e}))$. In this case, the area of the largest triangle is the one that does not contain $a$ or $b$. Thus, if we compute the square of the areas of the four triangle, we can detect this case too. 


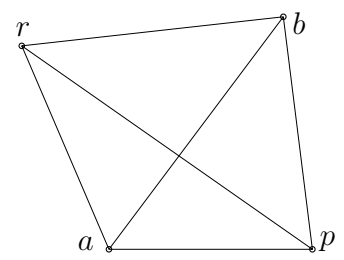

(a)

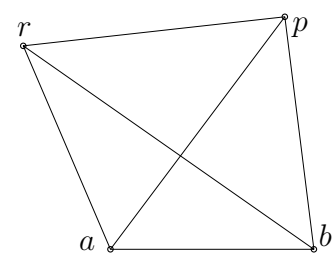

(b)

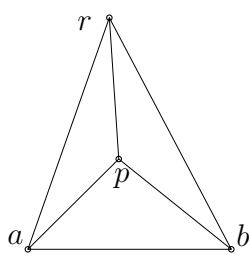

(c)

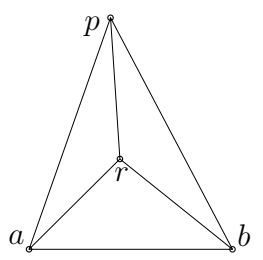

(d)

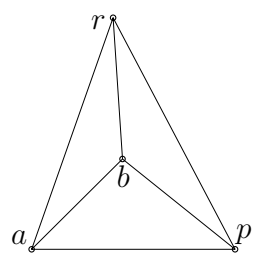

(e)

Figure 2: The five non-isomorphic configurations needed to consider for a quadrilateral on four points in Lemma 10

Assume that $G^{\prime}$ is a UDG and has an optimum clique partition of size $\alpha \leq \ell$. The cliques fall in two categories: small (having at most $2 \alpha-2$ points), and large (having at least $2 \alpha-1$ points). We focus only on finding the large cliques since it is easy to guess all the small cliques. Suppose for each pair $C_{i}, C_{j} \in \mathrm{OPT}$ of large cliques, we guess their respective representatives, $c_{i}$ and $c_{j}$. Further, suppose that we also guess a separating line $l_{i j}$ correctly which goes through points $u_{i j}$ and $v_{i j}$. For a point $p$ that is adjacent to $c_{i}$ or $c_{j}$ we want to efficiently test if $p$ is on the the same side of line $l_{i j}$ as $c_{i}$ (the positive side), or on $c_{j}$ 's side (the negative side), using only edge-lengths. Without loss of generality, let both $u_{i j}$ and $v_{i j}$ belong to clique $C_{i}$. For every node $p$ different from the representatives:

- Suppose $p$ is adjacent to all of $c_{i}, u_{i j}, v_{i j}, c_{j}$. Observe that we also have the edges $c_{i} u_{i j}$ and $c_{i} v_{i j}$. Given the edge-lengths of all the six edges among the four vertices $c_{i}, u_{i j}, v_{i j}, p$ using Lemma 10 we can decide if in a realization of these four points, the line going through $u_{i j}, v_{i j}$ separates the two points $p$ and $c_{i}$ or not. If $p$ and $c_{i}$ are on the same side, we say $p$ is on the positive side of $l_{i j}$ for $C_{i}$. Else, it is on the positive side of $l_{i j}$ for $C_{j}$.

- Suppose $p$ is adjacent to $c_{i}$ (and also to $u_{i j}$ and $v_{i j}$ ) but not to $c_{j}$. Given the edge-lengths of all the six edges among the four vertices $c_{i}, u_{i j}, v_{i j}, p$ using Lemma 10 we can decide if in a realization of these four points, the line going through $u_{i j}, v_{i j}$ separates the two points $p$ and $c_{i}$ or not. If $p$ and $c_{i}$ are on the same side, we say $p$ is on the positive side of $l_{i j}$ for $C_{i}$. Else, it is on the positive side of $l_{i j}$ for $C_{j}$.

For each $C_{i}$ and all the lines $l_{i j}$, consider the set of nodes that are on the positive side of all these lines with respect to $C_{i}$; we place these nodes in $C_{i}$. After obtaining the large and the small cliques, we obtain sets $C_{1}, \ldots, C_{\alpha}$. At the end we check if each $C_{i}$ forms a clique and if their union covers all the points. The number of guesses for representatives is $n^{O(\alpha)}$ and the number of guesses for the separator lines is $n^{O\left(\alpha^{2}\right)}$. So there are a total of $n^{O\left(\alpha^{2}\right)}$ configurations that we consider.

Clearly, if $G^{\prime}$ is a UDG then some set of guesses is a correct one, allowing us to obtain an optimum clique partition. If $G^{\prime}$ is not a UDG, we may still find a clique partition of $G^{\prime}$. However, if we fail to obtain a clique partition in our search then the subgraph is a certificate that $G^{\prime}$ is not a UDG. 


\section{$3 \quad(2+\varepsilon)$-Approximation for Weighted Clique Partition using Ad- jacency}

In this section we consider a generalization of the minimum clique partition on UDGs, which we call minimum weighted clique partition (MWCP). Given a node-weighted graph $G(V, E)$ with vertex weight wt $(v)$, the weight of a clique $C$ is defined as the weight of the heaviest vertex in it. For a clique partition $\mathcal{C}=\left\{C_{1}, C_{2}, \ldots, C_{t}\right\}$, the weight of $\mathcal{C}$ is defined as sum of the weights of the cliques in $\mathcal{C}$, i.e. wt $(\mathcal{C})=\mathbf{w t}\left(\bigcup_{i=1}^{t} C_{i}\right)=\sum_{i=1}^{t} \mathbf{w t}\left(C_{i}\right)$. The problem is, given $G$ in standard form, say, as an adjacency matrix, construct a clique partition $\mathcal{C}=\left\{C_{1}, C_{2}, \ldots, C_{t}\right\}$ while minimizing wt $(\mathcal{C})$. The weighted version of the problem as it is defined above has also been studied in different contexts. See [7, 3, 8, for study of weighted clique-partition on interval graphs and circular arc graphs.

Observe that MWCP distinguishes itself from MCP in two important ways: (i) The separability property which was crucially used earlier to devise a PTAS does not hold in the weighted case, and (ii) the number of cliques in an optimal solution for a UDG in a region of bounded radius is not bounded by the diameter of the region anymore, i.e. it is easy to construct examples of weighted UDGs in a bounded region where an optimal weighted clique partition contains an unbounded (in terms of region diameter) number of cliques. In addition, examples where two cliques in an optimal solution are not separable, that is, their convex hulls overlap, is easy to construct. (See the examples given in Figure 3.) To the best of our knowledge, MWCP has not been investigated before on UDGs. We, however, note that a simple modification to the algorithm by [18] also yields a factor- 8 approximation to the weighted case, a generalization which they do not consider. Here,

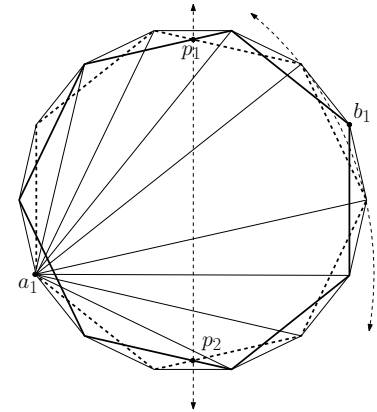

(a)

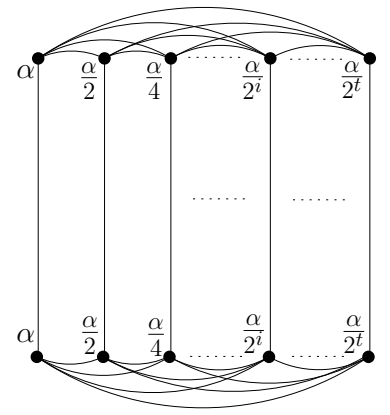

(b)

Figure 3: (a) Two overlapping weighted cliques, $A=\left\{a_{1}, \ldots, a_{k}\right\}$ and $B=\left\{b_{1}, \ldots, b_{k}\right\}$ are shown, $a_{i}, b_{i}$ are independent for all $i$. The heavy polygon has vertices weighted $k$ while the dashed ones are weighted 1 . opt $=k+1$ while any separable partition must pay a cost of at least $2 k$. (b) A UDG which is a matching between two cliques for which OPT contains $t$ cliques. The weight is less than $2 \cdot \alpha$.

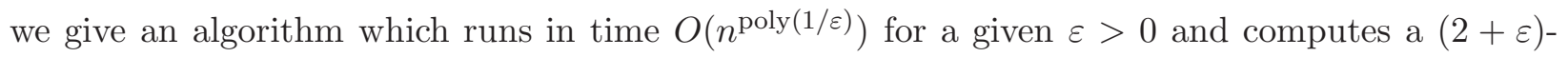
approximation to MWCP for UDGs expressed in standard form, for example, as an adjacency matrix. Our algorithm is weakly robust in that it either produces a clique partition or produces a polynomial-sized certificate proving that the input is not a UDG. When the input is a UDG, the algorithm returns a clique partition and it is guaranteed to be a $(2+\varepsilon)$-approximation; but if the input is not UDG there is no guarantee on the quality of the clique partition (if it computes one).

Theorem 11. Given a graph $G$ expressed in standard form, and $\varepsilon>0$, there is a polynomial 
time algorithm which either computes a clique partition of $G$ or gives a certificate that $G$ is not a $U D G$. If $G$ is a $U D G$, the weight of the clique partition computed is a $(2+\varepsilon)$-approximation of the minimum weighted clique partition (but there is no guarantee on the weight of the clique partition if the input graph is not $U D G$ ).

Our algorithm will borrow some ideas developed in Section 2 and in [18]. The high level idea of the algorithm is as follows. Similar to the algorithm in Section 2 , we first decompose the graph into bounded diameter regions and show that if we can compute a $(2+\varepsilon)$-approximate clique partition of each region then the union of these clique partitions is within $(2+\varepsilon)$ fraction of opt. We will employ a similar ball growing technique (as in Section 2) that will give us bounded diameter regions. We then show that we can either compute a clique partition or give a certificate that the subgraph is not a UDG. If the subgraph is a UDG, then our clique partition is within a factor $(2+\varepsilon)$ of the optimal. For the case of bounded diameter region, although the optimum solution may have a large number of cliques, we can show that there is a clique partition with small number of cliques whose cost is within $(1+\varepsilon)$-factor of the optimum solution. First we describe the main algorithm. Then in Subsection 3.1 we show that for each subgraph $B_{r}(v)$ (of bounded diameter) there is a near optimal clique partition with $\tilde{O}\left(r^{2}\right)$ cliques. Then in Subsection 3.2 we show how to find such a near optimal clique partition.

Let us denote the weight of the optimum clique partition of $G$ by opt. As before, let $B_{r}(v)=$ $\{u: d(u, v) \leq r\}$, called the ball of (unweighted) distance $r$ around $v$, be the set of vertices that are at most $r$ hops from $v$ in $G$. Our decomposition algorithm described below (see Algorithm 3) is similar to Algorithm 2 and partitions the graph into bounded diameter subgraphs below. The procedure CP, given a graph induced by the vertices of $B_{r}(v)$ and a parameter $\ell=\operatorname{poly}(r)$, runs in time $\left.n^{O\left(\ell^{2}\right)}\right)$ and either gives a certificate that $B_{r}(v)$ is not a UDG or computes a clique partition of $B_{r}(v)$; this clique partition is within a factor $(2+\varepsilon)$ of the optimum if $B_{r}(v)$ is a UDG. We only call this procedure for constant values of $r$. In the following, let $0<\gamma \leq \frac{\sqrt{9+4 \varepsilon}-3}{2}$ be a rational number. See Algorithm 3.

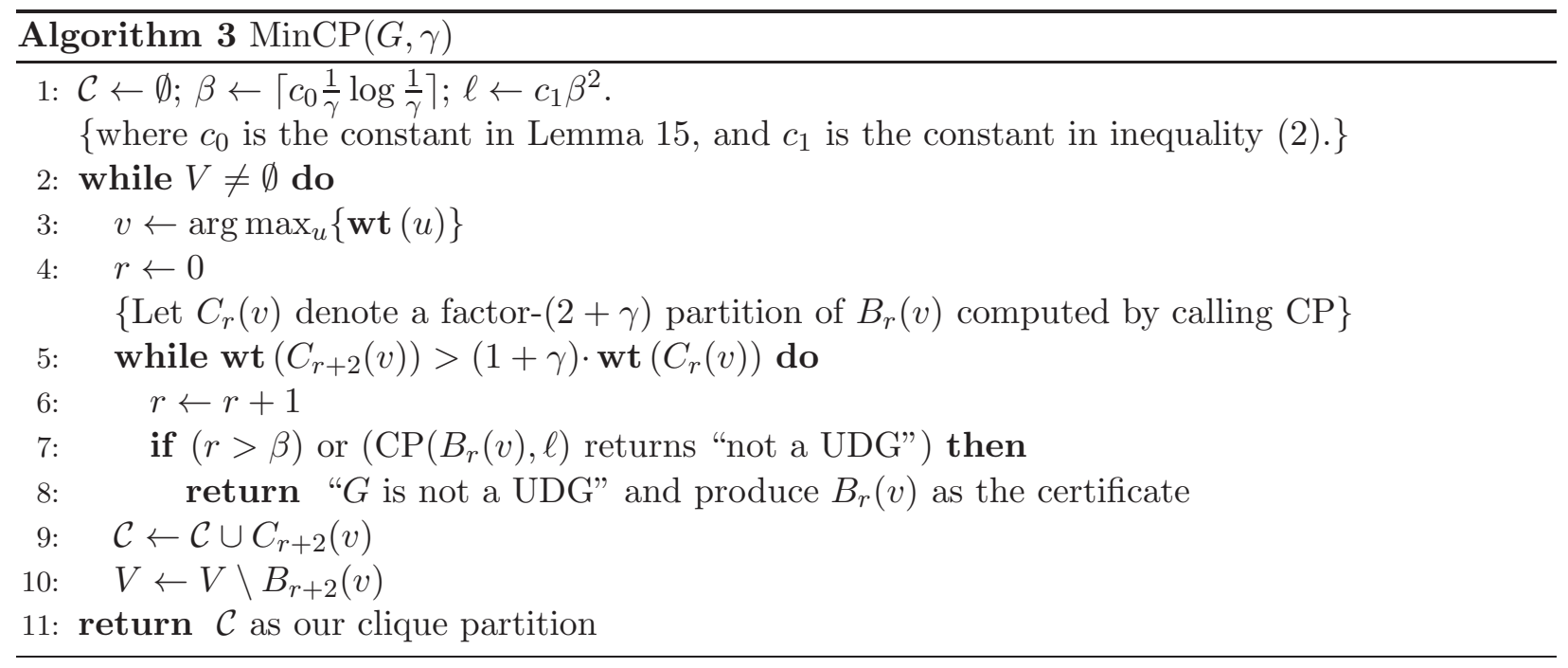

Let $k$ is the maximum number of iterations of the outer "while-loop". The proof of the following Lemma is identical to the proof of Lemma 6.

Lemma 12. Every two vertices $v \in B_{r_{i}^{*}}\left(v_{i}\right)$ and $u \in B_{r_{j}^{*}}\left(v_{j}\right)$ are non-adjacent. 
The following lemma shows a lower-bound for opt.

Lemma 13. $(2+\gamma) \cdot$ opt $\geq \boldsymbol{w} \boldsymbol{t}\left(\bigcup_{i=1}^{k} C_{r_{i}^{*}}\left(v_{i}\right)\right)$

Proof. Note that $B_{r_{i}^{*}}\left(v_{i}\right)$ is obtained by constructing a BFS tree rooted at vertex $v_{i}$ up to some depth $r_{i}^{*}$. Since the algorithm removes a super-set, $B_{r_{i}^{*}+2}\left(v_{i}\right)$, which has two more levels of the BFS tree, using the previous lemma there is no edge between any two nodes $v \in B_{r_{i}^{*}}\left(v_{i}\right)$ and $u \in B_{r_{j}^{*}}\left(v_{j}\right)$ for any pair $i \neq j$. So, no single clique in an optimum solution can contain vertices from distinct $B_{r_{i}^{*}}\left(v_{i}\right)$ and $B_{r_{j}^{*}}\left(v_{j}\right)$. Consider the subset of cliques in an optimal clique partition of $G$ that intersect $B_{r_{i}^{*}}\left(v_{i}\right)$ and call this subset $\mathrm{OPT}_{i}$. The argument above shows that $\mathrm{OPT}_{i}$ is disjoint from $\mathrm{OPT}_{j}$. Also, each $\mathrm{OPT}_{i}$ contains all the vertices in $B_{r_{i}^{*}}\left(v_{i}\right)$. Since $C_{r_{i}^{*}}\left(v_{i}\right)$ is a factor- $(2+\gamma)$ approximation for $B_{r_{i}^{*}}\left(v_{i}\right),(2+\gamma) \cdot \mathbf{w t}\left(\mathrm{OPT}_{i}\right) \geq \mathbf{w t}\left(C_{r_{i}^{*}}\left(v_{i}\right)\right)$. The lemma immediately follows by observing that $\mathrm{OPT}_{i}$ and $\mathrm{OPT}_{j}$ are disjoint.

We can relate the cost of our clique partition to opt as follows.

Lemma 14. If $\boldsymbol{w} \boldsymbol{t}\left(C_{r_{i}^{*}+2}\left(v_{i}\right)\right) \leq(1+\gamma) \boldsymbol{w} \boldsymbol{t}\left(C_{r_{i}^{*}}\left(v_{i}\right)\right)$, then $\boldsymbol{w} \boldsymbol{t}\left(\bigcup_{i=1}^{k} C_{r_{i}^{*}+2}\left(v_{i}\right)\right) \leq(2+\varepsilon)$ opt.

Proof.

$$
\mathbf{w} \mathbf{t}\left(\bigcup_{i=1}^{k} C_{r_{i}^{*}+2}\left(v_{i}\right)\right)=\sum_{i=1}^{k} \mathbf{w t}\left(C_{r_{i}^{*}+2}\left(v_{i}\right)\right) \leq(1+\gamma) \cdot \sum_{i=1}^{k} \mathbf{w} \mathbf{t}\left(C_{r_{i}^{*}}\left(v_{i}\right)\right) \leq(2+\gamma)(1+\gamma) \cdot \mathbf{o p t},
$$

where the last inequality uses Lemma 13 .

Next, we show that the inner "while-loop" terminates in $\tilde{O}\left(\frac{1}{\gamma}\right)$, that is each $r_{i}^{*}$ is bounded by $\tilde{O}\left(\frac{1}{\gamma}\right)$. This is similar to the proof of Lemma 9 . Since the while loop terminates, $r_{i}^{*}$ exists and by definition of $r_{i}^{*}$, it must be the case that for all smaller values of $r<r_{i}^{*}$, wt $\left(C_{r}\left(v_{i}\right)\right)>$ $(1+\gamma) \cdot \mathbf{w t}\left(C_{r-2}\left(v_{i}\right)\right)$. Because the diameter of $B_{r}\left(v_{i}\right)$ is $O(r)$, if $B_{r}(v)$ is a UDG, there is a realization of it in which all the points fit into a $r \times r$ grid. Also, since $v_{i}$ is a heaviest vertex in the (residual) graph, there is a clique partition whose weight is at most $\alpha \cdot \mathbf{w t}\left(v_{i}\right) \cdot r^{2}$. Therefore, wt $\left(C_{r}\left(v_{i}\right)\right)<\alpha \cdot \mathbf{w t}\left(v_{i}\right) \cdot r^{2}$, for some constant $\alpha$. So:

$\alpha \cdot \mathbf{w t}\left(v_{i}\right) \cdot r^{2}>\mathbf{w t}\left(C_{r}\left(v_{i}\right)\right)>(1+\gamma) \cdot \mathbf{w t}\left(C_{r-2}\left(v_{i}\right)\right)>\ldots>(1+\gamma)^{\frac{r}{2}} \cdot \mathbf{w t}\left(C_{0}\left(v_{i}\right)\right)=\mathbf{w t}\left(v_{i}\right) \cdot(\sqrt{1+\gamma})^{r}$,

which implies $\alpha \cdot r^{2}>(\sqrt{1+\gamma})^{r}$, for the case that $r$ is even. If $r$ is odd we obtain $\alpha \cdot r^{2}>$ $(\sqrt{1+\gamma})^{r-1}$. Thus, the following lemma easily follows:

Lemma 15. There is a constant $c_{0}>0$ such that for each $i: r_{i}^{*} \leq c_{0} / \gamma \cdot \log 1 / \gamma$.

In Subsection 3.2, we show the algorithm CP that given $B_{r}(v)$ and an upper bound $\ell$ on $\left|C_{r}(v)\right|$, either computes a clique partition (which is within a factor $2+\gamma$ of opt if $B_{r}(v)$ is a UDG) or detects that the graph is not UDG; the algorithm runs in time $n^{O\left(\ell^{2}\right)}$. By the above arguments, if $B_{r}(v)$ is a unit disk graph then there is a constant $c_{1}>0$ such that:

$$
\left|C_{r}(v)\right|=O\left(r_{i}^{* 2}\right) \leq c_{1} \cdot \frac{c_{0}^{2}}{\gamma^{2}} \log ^{2} \frac{1}{\gamma}
$$


We can set $\ell=\left\lceil c_{1} \frac{c_{0}^{2}}{\gamma^{2}} \log ^{2} \frac{1}{\gamma}\right\rceil$ for any invocation of OPT-CP as an upper bound, where $c_{1}$ is the constant in $O\left(r_{i}^{* 2}\right)$. So, the running time of the algorithm is $n^{\tilde{O}\left(1 / \varepsilon^{4}\right)}$.

\subsection{Existence of a Small Clique Partition of $B_{r}(v)$ having Near-optimal Weight}

Unlike the unweighted case, an optimal weighted clique partition in a small region may contain a large number of cliques. Yet, there exists a partition whose weight is within a factor $\left(1+\frac{\gamma}{2}\right)$ of the minimum weight which contains few cliques (where by "few" we mean $\ell$ as in Algorithm 3). The existence of a light and small partition allows us to enumerate them in the same manner in the algorithm of subsection 2.3, yielding a $(2+\gamma)$-approximation for the problem instance in a ball of small radius. In the following, let $r \in \tilde{O}\left(\frac{1}{\gamma}\right)$; we focus on the subproblem that lies in some $B_{r}(v)$. Recall that any ball of radius $r$ can be partitioned into $O\left(r^{2}\right)$ cliques (Lemma 3). We begin with a simple lemma which states that for any clique partition $\mathcal{C}$, if the set of vertices can be be covered by another clique partition $\mathcal{C}^{\prime}$ containing $x$ cliques then the sum of the weights of the $x$ cliques in $\mathcal{C}^{\prime}$ is not significantly more than the weight of the heaviest clique in $\mathcal{C}$.

Lemma 16. For any collection of disjoint cliques $\mathcal{C}=\left\{C_{1}, C_{2}, \ldots, C_{t}\right\}$ having weights such that $\boldsymbol{w} \boldsymbol{t}\left(C_{1}\right) \geq \boldsymbol{w} \boldsymbol{t}\left(C_{2}\right) \geq \ldots \geq \boldsymbol{w} \boldsymbol{t}\left(C_{t}\right)$ suppose the vertices of $\mathcal{C}$ can be partitioned into $x$ cliques $\mathcal{C}^{\prime}=\left\{C_{1}^{\prime}, C_{2}^{\prime}, \ldots, C_{x}^{\prime}\right\}$. Then $\boldsymbol{w t}\left(\mathcal{C}^{\prime}\right)=\boldsymbol{w} \boldsymbol{t}\left(\bigcup_{l=1}^{x} C_{l}^{\prime}\right)=\sum_{l=1}^{x} \boldsymbol{w t}\left(C_{l}^{\prime}\right) \leq x \cdot \boldsymbol{w} \boldsymbol{t}\left(C_{1}\right)$

Proof. Without loss of generality, let wt $\left(C_{1}^{\prime}\right) \geq \mathbf{w t}\left(C_{2}^{\prime}\right) \geq \ldots \geq \mathbf{w t}\left(C_{x}^{\prime}\right)$. Since $\mathcal{C}^{\prime}$ partitions vertices in $\mathcal{C}$, wt $\left(C_{1}^{\prime}\right)=\mathbf{w t}\left(C_{1}\right)$. Since $\left|\mathcal{C}^{\prime}\right|=x$, wt $\left(\mathcal{C}^{\prime}\right)=\sum_{l=1}^{x} \mathbf{w t}\left(C_{l}^{\prime}\right) \leq x \cdot \mathbf{w t}\left(C_{1}^{\prime}\right)=x \cdot \mathbf{w t}\left(C_{1}\right)$.

In an optimal partition of a ball of radius $r$, the sum of the weights of the lighter cliques is not significantly more than its weight.

Lemma 17. Let $\mathcal{C}=\left\{C_{1}, C_{2}, \ldots, C_{t}\right\}$ be an optimal clique partition and let $\boldsymbol{w t}\left(C_{1}\right) \geq \boldsymbol{w} \boldsymbol{t}\left(C_{2}\right) \geq$ $\ldots \geq \boldsymbol{w t}\left(C_{t}\right)$. Suppose there is another clique partition $\mathcal{C}^{\prime}=\left\{C_{1}^{\prime}, \ldots, C_{x}^{\prime}\right\}$ of the vertices of $\mathcal{C}$. Then, for every $1 \leq i<t$ : $(x-1) \cdot \boldsymbol{w t}\left(C_{i}\right) \geq \sum_{l=i+1}^{t} \boldsymbol{w} \boldsymbol{t}\left(C_{l}\right)$.

Proof. By way of contradiction, suppose there exists an index $1 \leq j<t$ such that $(x-1) \cdot \mathbf{w t}\left(C_{j}\right)<$ $\sum_{l=j+1}^{t} \mathbf{w t}\left(C_{l}\right)$. Because $\bigcup_{l=1}^{t} C_{l}$ can be covered by $\mathcal{C}^{\prime}$, so can $\bigcup_{l=j}^{t} C_{l}$. Let the $2 \leq x^{\prime} \leq x$ be the smallest index such that $\mathcal{C}_{j}^{\prime}=\left\{C_{1}^{\prime}, C_{2}^{\prime}, \ldots, C_{x^{\prime}}^{\prime}\right\}$ covers $\bigcup_{l=j}^{t} C_{l}$. On the other hand, $\left(x^{\prime}-\right.$ $1) \cdot \mathbf{w t}\left(C_{j}\right) \leq(x-1) \cdot \mathbf{w t}\left(C_{j}\right)<\sum_{l=j+1}^{t} \mathbf{w t}\left(C_{l}\right)$, which implies

$$
\text { opt }=\sum_{l=1}^{t} \mathbf{w t}\left(C_{l}\right)>\sum_{l=1}^{j} \mathbf{w t}\left(C_{l}\right)+\left(x^{\prime}-1\right) \cdot \mathbf{w t}\left(C_{j}\right)=\sum_{l=1}^{j-1} \mathbf{w t}\left(C_{l}\right)+x^{\prime} \mathbf{w} \mathbf{t}\left(C_{j}\right) .
$$

By Lemma 16, wt $\left(\mathcal{C}_{j}^{\prime}\right) \leq x^{\prime} \cdot \mathbf{w t}\left(C_{j}\right)$. This, combined with inequality (3) implies opt $>\sum_{l=1}^{j-1} \mathbf{w t}\left(C_{l}\right)+$ $\sum_{l=1}^{x^{\prime}} \mathbf{w t}\left(C_{l}^{\prime}\right)$. Therefore the cliques in $\mathcal{C}^{\prime \prime}=\left\{C_{1}, C_{2}, \ldots, C_{j-1}, C_{1}^{\prime}, C_{2}^{\prime}, \ldots, C_{x^{\prime}}^{\prime}\right\}$ cover all the nodes of cliques in $\mathcal{C}$ and has cost smaller than opt. If a vertex belongs to two or more cliques in $\mathcal{C}^{\prime \prime}$ we remove it from all but one of them to obtain a clique partition with cost no more than cost of $\mathcal{C}^{\prime \prime}$ which is smaller than opt. This completes the proof. 
We now are ready to prove the main result of this section which states that for any optimal weighted clique partition of a ball of radius $r$, there exists another clique partition whose weight is arbitrarily close to the weight of the optimal partition, but has $O\left(r^{2}\right)$ cliques in it. Since the radius of the ball within which the subproblem lies is small, $r \in \tilde{O}\left(\frac{1}{\gamma}\right)$, this means that if we were to enumerate all the clique partitions of the subproblem up to $O\left(r^{2}\right)$, we will see one whose weight is arbitrarily close to the weight of an optimal clique. Choosing a lightest one from amongst all such cliques guarantees that we will choose a one whose weight is arbitrarily close to the optimal weight.

Lemma 18. Let $\gamma>0$ and $r \in \tilde{O}(1 / \gamma)$ be two constants. Let $\mathcal{C}=\left\{C_{1}, C_{2}, \ldots, C_{t}\right\}$ be an optimal weighted clique partition of $B_{r}(v)$ and let $\mathcal{C}^{\prime}=\left\{C_{1}^{\prime}, \ldots, C_{x}^{\prime}\right\}$ be another clique partition of vertices of $\mathcal{C}$ with $x \in O\left(r^{2}\right)$. Let $\boldsymbol{w} \boldsymbol{t}\left(C_{1}\right) \geq \boldsymbol{w} \boldsymbol{t}\left(C_{2}\right) \geq \ldots \geq \boldsymbol{w} \boldsymbol{t}\left(C_{t}\right)$. Then, there is a partition of vertices of $\mathcal{C}$ into at most $j+x$ cliques for some constant $j=j(\gamma)$, with cost at most $\left(1+\frac{\gamma}{2}\right)$ opt.

Proof. Without loss of generality, we assume that both $x$ and $t$ are at least two (as if $B_{r}(v)$ is a clique we are done). Consider an arbitrary value of $j \leq t$. Since $\bigcup_{l=1}^{t} C_{l}$ can be covered by $x$ cliques in $\mathcal{C}^{\prime}$, there is an index $x^{\prime}\left(2 \leq x^{\prime} \leq x\right)$ such that $\bigcup_{l=j}^{t} C_{l}$ can be covered by $\mathcal{C}_{j}^{\prime}=\left\{C_{1}^{\prime}, C_{2}^{\prime}, \ldots, C_{x^{\prime}}^{\prime}\right\}$. By applying Lemma 17 repeatedly:

$$
\begin{gathered}
\text { opt } \geq \sum_{l=1}^{j} \mathbf{w t}\left(C_{l}\right) \geq \frac{1}{x^{\prime}-1}\left(\sum_{l=2}^{j} \mathbf{w t}\left(C_{l}\right)\right)+\sum_{l=2}^{j} \mathbf{w t}\left(C_{l}\right) \geq \ldots \geq\left(\frac{x^{\prime}}{x^{\prime}-1}\right)^{j-1} \cdot \mathbf{w t}\left(C_{j}\right) \\
\Rightarrow \operatorname{opt} \frac{\left(x^{\prime}-1\right)^{j-1}}{x^{\prime j-2}} \geq x^{\prime} \cdot \mathbf{w t}\left(C_{j}\right)
\end{gathered}
$$

Using inequality (4):

$$
\text { opt }+\frac{\text { opt } \cdot\left(x^{\prime}-1\right)^{j-1}}{x^{\prime j-2}} \geq \sum_{l=1}^{j-1} \mathbf{w t}\left(C_{l}\right)+x^{\prime} \cdot \mathbf{w t}\left(C_{j}\right) \geq \sum_{l=1}^{j-1} \mathbf{w t}\left(C_{l}\right)+\sum_{l=1}^{x^{\prime}} \mathbf{w t}\left(C_{l}^{\prime}\right),
$$

where the second inequality follows by applying Lemma 16. Let $\mathcal{C}^{\prime \prime}=\left\{C_{1}, \ldots, C_{j-1}, C_{1}^{\prime}, \ldots, C_{x^{\prime}}^{\prime}\right\}$. Thus, the cliques in $\mathcal{C}^{\prime \prime}$ cover all the vertices of $\mathcal{C}$ and has total cost at most $\left(1+\frac{\left(x^{\prime}-1\right)^{j-1}}{x^{\prime j-2}}\right)$ opt by inequality (5). If a vertex belongs to two or more cliques in $\mathcal{C}^{\prime \prime}$ we remove it from all but one of them arbitrarily to obtain a clique partition of size $j-1+x^{\prime}$ and whose total cost is upper bounded by $\left(1+\frac{\left(x^{\prime}-1\right)^{j-1}}{x^{\prime j-2}}\right)$ opt. Note that, $\frac{\left(x^{\prime}-1\right)^{j-1}}{x^{\prime j-2}}=\left(x^{\prime}-1\right)\left(\frac{x^{\prime}-1}{x^{\prime}}\right)^{j-2}$ and $0<\frac{x^{\prime}-1}{x^{\prime}}<1$ (because $\left.x^{\prime} \geq 2\right)$.

Since $r \in \tilde{O}\left(\frac{1}{\gamma}\right)$ and $x^{\prime} \leq x \in O\left(r^{2}\right)$, for an appropriate choice of $j=j(\gamma),\left(x^{\prime}-1\right)\left(\frac{x^{\prime}-1}{x^{\prime}}\right)^{j-2}<\gamma / 2$. Thus we obtain a clique partition with $j+x-1$ cliques and cost at most $(1+\gamma / 2) \cdot$ opt. This proves the lemma.

\section{$3.2(2+\gamma)$-Approximation for MWCP in $B_{r}(v)$}

Finally, we show how to compute a $(2+\gamma)$-approximate MWCP of the graph $B_{r}(v)$ for any given $\gamma$. For an edge ordering $L=\left(e_{1}, e_{2}, \ldots, e_{m}\right)$ of a graph $G$ with $m$ edges, let $G_{L}[i]$ denote the edge induced subgraph with edge-set $\left\{e_{i}, e_{i+1}, \ldots, e_{m}\right\}$. For each $e_{i}$, let $N_{L}[i]$ denote the common neighborhood of the end-points of $e_{i}$ in $G_{L}[i]$. An edge ordering $L=\left(e_{1}, e_{2}, \ldots, e_{m}\right)$ is a CNEEO if 
for every $e_{i}$ in $L, N_{L}(i)$ induces a co-bipartite graph in $G$. It is known [20] that every UDG graph admits a co-bipartite edge elimination ordering (CNEEO). In the following, let $G_{v}$ denote $B_{r}(v)$. We state a lemma of [18].

Lemma 19. [18] Let $C$ be a clique in $G_{v}$, and let $L$ be a CNEEO of $G_{v}$. Then, there is an $i$, $1 \leq i \leq m$, such that $N_{L}[i]$ contains $C$.

Assume that $G_{v}$ can be partitioned into $\alpha \leq \ell=\tilde{O}\left(1 / \gamma^{2}\right)$ cliques, $\mathcal{O}=\left\{O_{1}, O_{2}, \ldots, O_{\alpha}\right\}$, such that $\mathbf{w t}(\mathcal{O}) \leq\left(1+\frac{\gamma}{2}\right) \cdot \mathbf{w t}\left(\mathrm{OPT}_{v}\right)$, where $\mathrm{OPT}_{v}$ is an optimal weighted clique partition of $G_{v}$. Note that by Lemma 18 this is true for subgraph $B_{r}(v)$. Suppose that we are given the upper bound $\ell$; we will try all possible values of $\alpha$. Without loss of generality, let wt $\left(O_{1}\right) \geq \mathbf{w t}\left(O_{2}\right) \geq \ldots \geq \mathbf{w t}\left(O_{\alpha}\right)$. Observe that, without loss of generality, we can assume $O_{i}$ is a maximal clique in $\bigcup_{j=i}^{\alpha} O_{j}$. The implication of the above lemma is that even though we do not know $O_{1}$, hence we do not know $\mathcal{O}$, we do know that for every CNEEO $L$ of $G_{v}$, there is an $e_{i}$ such that $N_{L}[i]$ can be partitioned into at most two cliques that fully cover $O_{1}$. Since $O_{1}$ is a heaviest clique, the two cliques that cover the subgraph $N_{L}[i]$ pay a cost of at most $2 \cdot \mathbf{w t}\left(O_{1}\right)$. This suggests an algorithm that guesses an edge sequence $\left(f_{1}, f_{2}, \ldots, f_{\alpha}\right)$ of $G_{v}$. Then, the algorithm computes $L$, a CNEEO of $G_{v}$. The algorithm's first guess is "good" if $f_{1}$ is an edge in $O_{1}$ that occurs first in $L$. Suppose that this is the case and suppose that $f_{1}$ has rank $i$ in $L$. Then, $O_{1}$ is contained in $N_{L}[i]$, and we cover $N_{L}[i]$ with at most two cliques. Call these $C_{1}^{\prime}$ and $C_{1}^{\prime \prime}$ and wt $\left(C_{1}^{\prime}\right)+\mathbf{w t}\left(C_{1}^{\prime \prime}\right) \leq 2 \cdot \mathbf{w t}\left(O_{1}\right)$. So, when we remove $N_{L}[i]$ from $G_{v}$, we get a UDG which can be partitioned into at most $\alpha-1$ cliques, namely, $\mathcal{O}^{\prime}=\left\{O_{2}, \ldots, O_{\alpha}\right\}$. We then again construct a CNEEO, $L^{\prime}$, of $G_{v}^{\prime}=G_{v} \backslash N_{L}[i]$. Just like before, our guess $f_{2}$ is "good" if $f_{2}$ is an edge in $O_{2}$ and occurs first in $L^{\prime}$. Let $i^{\prime}$ be the rank of $f_{2}$ in $L^{\prime}$, we see that $N_{L^{\prime}}\left[i^{\prime}\right]$ fully contains $O_{2}$, and we again cover it with at most 2 cliques. Next, delete $N_{L^{\prime}}\left[i^{\prime}\right]$ from $G_{v}^{\prime}$ to get a graph which can be partitioned into $\alpha-2$ cliques, and so on. See Algorithm 4 for details.

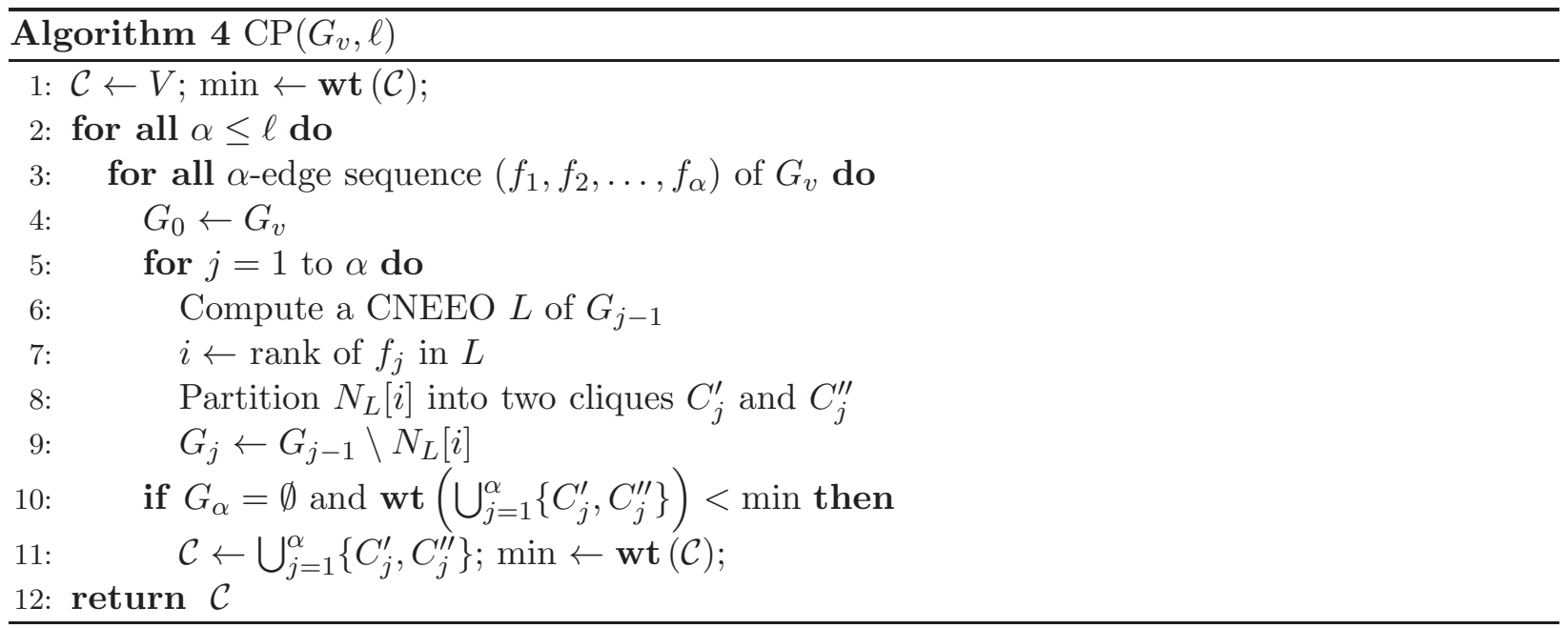

Note that while Lemma 19 allows us to cover any clique with at most 2 cliques, it does not find the clique. In the algorithm, note that if at any point, the algorithm is unable to construct a CNEEO, we can declare that the graph $G_{v}$ is not a UDG. Also, if for all invocations of the algorithm by an external algorithm that guesses the value of opt we are unable to find a clique partition, then again we can declare that $G_{v}$ is not a UDG. 


\section{$4 O\left(\log ^{*} n\right)$-round Distributed PTAS for UDGs with Edge-Lengths}

In this section, we give details of a distributed PTAS for MCP which runs in $O\left(\frac{\log ^{*} n}{\varepsilon^{O(1)}}\right)$ rounds of distributed computation under the $\mathcal{L O C} \mathcal{A L}$ model of computation [16]. The model of computation that we employ assumes a synchronous system where communication between neighboring nodes takes place in synchronous rounds using messages of unbounded size [16]. So, in a single round of communication, any node acquires the subgraph (information pertaining to the set of nodes, edges, the states of local variables, etc.) within its immediate neighborhood. So, after $k$ rounds of communication, any node acquires complete knowledge about its $k$-neighborhood.

Observe that in Algorithm MinCP2, the radius $r$ of any ball $B_{v}(r)$ is bounded above by $\tilde{O}(1 / \varepsilon)$, while the center, $v$, is an arbitrary vertex. Since the radius of any ball is "small", the maximum number of rounds of distributed computation that the sequential algorithm needs before terminating the "while-loop" is also "small". Therefore, for any pair of balls $B_{u}\left(r_{i}\right)$ and $B_{v}\left(r_{j}\right)$, such that $d(u, v) \in \omega(1 / \varepsilon)$, one should be able to run part of the sequential algorithm in parallel, as they surely are independent of each other. We borrow some ideas from [10] and find regions that are far apart such that we can run the sequential algorithm in those regions in parallel. See Algorithm 5 for details.

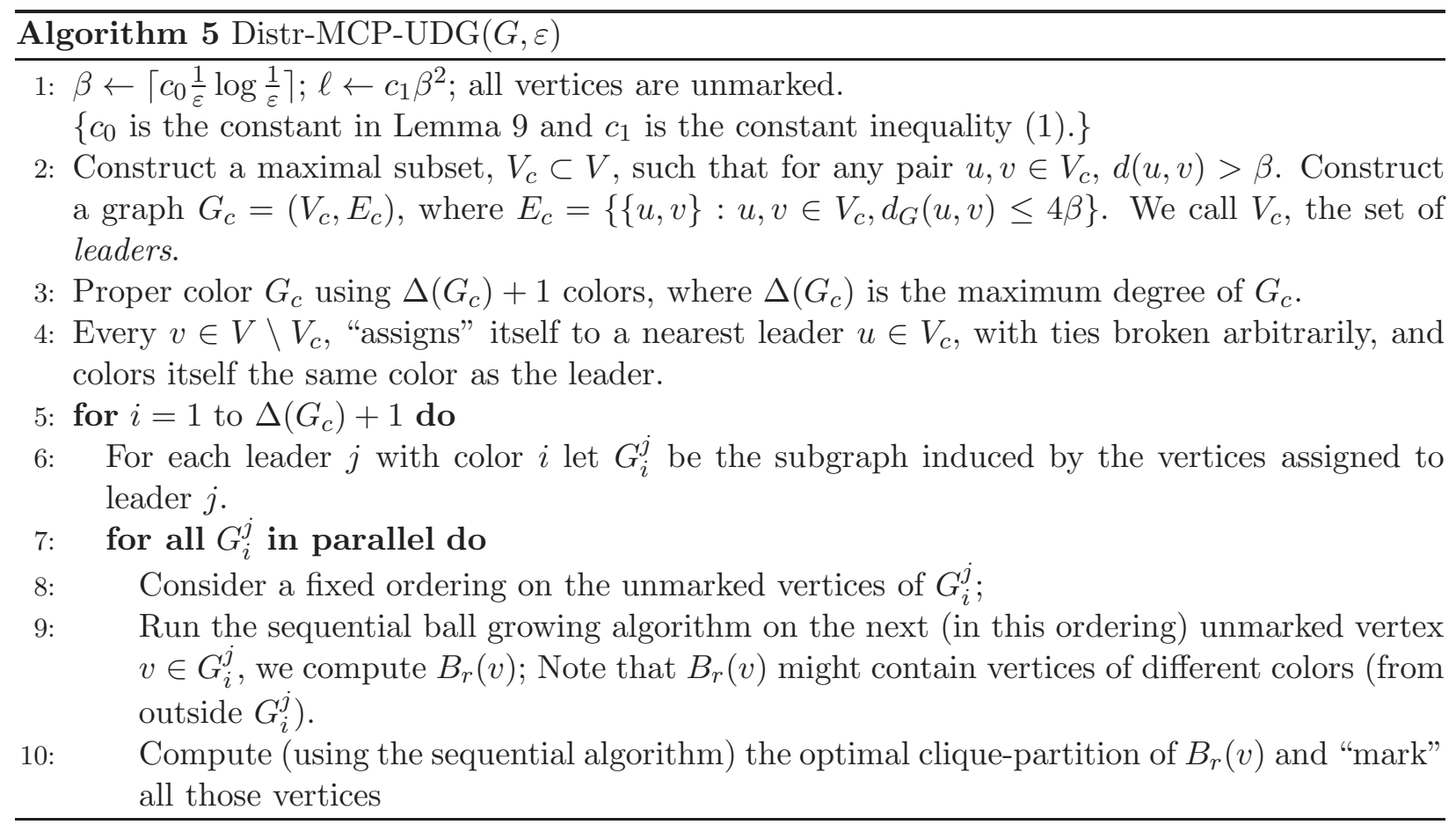

It should be pointed out that adapting the algorithm of [10] for maximum independent set and minimum dominating set to our setting is not trivial. The reason is that MCP is a partition of the entire vertex set and partitioning just a subset well enough will not do. Specifically [10] chooses a subset of vertices upon which their ball-growing algorithm is run; it suffices for their purposes to dispense with the remaining subset of vertices that were not picked by their ball-growing algorithm. If we had followed a similar scheme then we would surely get a good clique partition on a subset of vertices; however, it is unclear as to how to obtain a good partition of the remaining subset in 
terms of the optimal size for the original problem instance over the entire vertex set. As a means to circumvent this issue, we first construct a "crude" partition of the vertex set, instead of just a subset of vertices as done in [10].

\subsection{Analysis}

We now show that the algorithm constructs a $(1+\varepsilon)$-approximation to MCP on UDGs given only rational edge-lengths in $O\left(\log ^{*} n\right)$ rounds of distributed computation under the $\mathcal{L O C} \mathcal{A L}$ model; we first show correctness of the algorithm, followed by bounding the number of communication rounds.

Correctness: We prove that our algorithm is correct by showing that any execution of DistrMCP-UDG can be turned into a sequential execution of MinCP2. As stated, every vertex has the same color as its leader; let a leader vertex be its own leader. We first show that the distance of every vertex to its leader is small.

Lemma 20. For any vertex $v \notin V_{c}$, there is a vertex $u \in V_{c}$ such that $d_{G}(u, v) \leq \beta$.

Proof. Suppose not. So there is a $v$ whose distance to every $u \in V_{c}$ is more than $\beta$. But then $V_{c}^{\prime}=V_{c} \cup\{v\}$ has the property that for all $x, y \in V_{c}^{\prime}, d_{G}(x, y)>\beta$, contradicting the maximality of $V_{c}$.

Next we show that for any pair of vertices $u, v$ of the same color but with different leaders, the minimum distance between them is large enough so that a pair of balls of radius at most $\beta$ over them will be disjoint, where $\beta$ is defined in MinCP2 and Distr-MCP-UDG.

Lemma 21. Consider two leaders $x, y$ of the same color, say $i$, and any two vertices $u \in G_{i}^{x}$ and $v \in G_{i}^{y}$ (note that we might have $u=x$ or $v=y$ ). Then for all values of $r$ considered in the ball growing algorithm, $B_{r}(u)$ and $B_{r}(v)$ are disjoint.

Proof. Since $x$ and $y$ have the same color $d(x, y)>4 \beta$. By Lemma 20, any vertex in either of $G_{i}^{x}$ or $G_{i}^{y}$ is at a distance of at most $\beta$ from the respective leader; so, $d_{G}(u, v)>2 \beta$. The lemma follows easily by noting the fact that $r \leq \beta$ in the ball growing algorithm.

We are now ready to prove the correctness of Distr-MCP-UDG by showing an equivalence between any execution of it to some execution of MinCP2.

Lemma 22. Any execution of Distr-MCP-UDG from "Step 5" to "Step 10" can be converted to a valid execution of MinCP2.

Proof. Consider an arbitrary execution of Distr-MCP-UDG. Suppose that $V_{1}, V_{2}, V_{3}, \ldots$ is a sequence of disjoint sets of the vertices of $V$ such that we run the ball growing algorithm in parallel (during Distr-MCP-UDG) on vertices of $V_{1}$ (and thus we compute an optimal clique partition on each vertex of $V_{1}$ in parallel) then we do this for vertices in $V_{2}$, and so on. Note that the vertices in $V_{i}$ all have the same color and each has a different leader. Consider an arbitrary ordering $\pi_{i}$ of the vertices in each $V_{i}$ and suppose that we run MinCP2 algorithm on vertices of $V_{1}$ based on ordering $\pi_{1}$, then on vertices of $V_{2}$ based on ordering $\pi_{2}$, and so on. Since the vertices in each $V_{i}$ have distinct leaders, by Lemma 21, the balls grown around them are disjoint. It should be easy to see that the balls grown by algorithm MinCP2 is exactly the same as the ones computed by Distr-MCP-UDG. 
The following result follows immediately as a corollary to Lemma 22 .

Corollary 23. Given an $\varepsilon>0$, Distr-MCP-UDG constructs a clique partition of the input graph $G$ with associated edge-lengths, or produces a certificate that $G$ is not a UDG. If $G$ is a UDG then the size of the partition is within $(1+\varepsilon)$ of the optimum clique partition.

Running Time: We now show that the algorithm runs in $O\left(\frac{\log ^{*} n}{\varepsilon^{O(1)}}\right)$ distributed rounds under the $\mathcal{L O C} \mathcal{A} \mathcal{L}$ model of computation.

Lemma 24. "Step 2" requires $O\left(\beta \cdot \log ^{*} n\right)$ rounds of communication.

Proof. Observe that the result of "Step 2" is identical to constructing a maximal independent set (MIS) in $G^{\beta}$. Note that $G^{\beta}$ is also a UDG where the new unit is $\beta$. As a result, $G^{\beta}$ is a subclass of growth-bounded graphs [9] where all the distances are scaled by $\beta$; computation of MIS on $G^{\beta}$ takes $O\left(\beta \cdot \log ^{*} n\right)$ rounds [21] while the construction of $G^{\beta}$ takes $\beta$ rounds. Hence, the number of rounds needed by "Step 2" can be bounded by $O\left(\beta \cdot \log ^{*} n\right)$.

It is easy to see that constructing $G_{c}$ requires at most $4 \beta$ communication rounds. Next, we show that the maximum degree of $G_{c}, \Delta\left(G_{c}\right)$ is bounded by a constant.

Lemma 25. $\Delta\left(G_{c}\right) \in O(1)$

Proof. Let $v$ be a vertex of $G_{c}$ having maximum degree. Note that all its neighboring vertices in $G_{c}$ lie in a disk of radius at most $4 \beta$. Also note that due to "Step 2" the minimum distance between any pair of vertices in $G_{c}$ is more than $\beta$. As a result, any disk of diameter $\beta$ contains at most 1 vertex of $G_{c}$. Using standard packing arguments of the underlying space, a crude upper bound on the number of vertices of $G_{c}$ in a disk of radius at most $4 \beta$ is 256 vertices; this also upper bounds the degree of $v$.

Next, we bound the number of rounds needed for "Step 3"

Lemma 26. "Step 3" requires $O\left(\beta \cdot \log ^{*} n\right)$ rounds of communication.

Proof. For graphs whose maximum degree is $\Delta$, a $\Delta+1$ proper coloring requires $O\left(\Delta+\log ^{*} n\right)$ rounds [11. Since $\Delta\left(G_{c}\right) \in O(1)$ (Lemma 25), and the fact that distances in $G_{c}$ are scaled by a factor of $4 \beta$ as compared to the distances in $G$, a $\Delta\left(G_{c}\right)+1$ proper coloring of $G_{c}$ can be obtained in $O\left(\beta \cdot \log ^{*} n\right)$ rounds.

"Step 4" requires at most $\beta$ rounds of communication; according to Lemma 20, for every $v \notin V_{c}$, there is some $u \in V_{c}$ that is at a distance at most $\beta$ from it. The identity and color of such a vertex can be obtained in $\beta$ rounds. We can now bound the number of rounds that Distr-MCP-UDG requires. First, note that for any iteration, $i$, of "Step 7", only knowledge of a subgraph up to radius $\beta$ is required, and any node can obtain knowledge of the subgraph up to radius $\beta$ from it in $\beta$ rounds of communication. So, for any vertex in $G_{i}^{j}$ obtains knowledge about the "marked/unmarked" status of all the vertices in $G_{i}^{j}$ in $\beta$ rounds of communication. Since the diameter of each $G_{i}^{j}$ is at most $2 \beta$, the number of balls to grow in "Step 9." is at most $O\left(\beta^{2}\right)$. Therefore:

Theorem 27. Distr-MCP-UDG requires $O\left(\beta \cdot \log ^{*} n\right)$ rounds of communication under the $\mathcal{L} \mathcal{O C} \mathcal{A} \mathcal{L}$ model of computation. 


\section{Concluding Remarks}

Recall that the weakest assumption that we needed to obtain a PTAS for unweighted clique partition problem was that all the edge lengths are given. This information was crucially used in obtaining a robust PTAS. In the case of weighted clique partition, we gave a $(2+\varepsilon)$-approximation algorithm without the use of edge-lengths (using only the adjacency information). It will be interesting to see if a PTAS exists for the unweighted case but with reliance only on adjacency.

It is also unclear if a PTAS is possible even with the use of geometry in the weighted case. Recall that the PTAS given in Sections 2 crucially uses the idea of separability of an optimal clique partition. However, in the weighted case, even though a near optimal clique partition in a small region has few cliques, there are examples where any separable partition pays a cost at least factor- 2 to that of a near optimal partition. We give an example in Figure 3(a). In the example shown in Figure 3(a) two cliques of optimal weight are shown: one of them, $A$, whose vertices are the vertices of the $k$-gon shown in dashed-heavy lines, and the other, $B$, whose vertices are the vertices of the $k$-gon shown in solid-heavy lines. The example is that for $k=7$. The vertices of $A$ are labeled $a_{1}, a_{2}, \ldots, a_{k}$ in a counter-clockwise fashion. The vertices of $B$ are labeled such that $b_{i}$ is diametrically opposite to $a_{i}$. The distance between $a_{i}$ and $b_{i}$ is more than 1 while the distance between $a_{i}$ and $b_{j}, i \neq j$ is at most 1 . So, there is an edge between $a_{i}$ to every $a_{l}$ and to every $b_{j}, j \neq i$. This is also the case for $b_{i}$. In the figure, the edges incident to $a_{1}$ are shown by solid-light lines. Also, the dashed arc shows part of the unit disk boundary that is centered at $a_{1}$ - note that it does not include $b_{1}$. Let the weights of vertices in $A$ be $k$ and the weights of vertices in $B$ be 1 . Clearly, opt $\leq k+1$. However, any separable clique partition pays a cost of at least $2 k$ : if vertices in $A$ must all belong to a common clique, then every vertex in $B$ must belong to a distinct clique in a separable clique partition. Also, note that as-per separability, a line going through $\left\{p_{1}, p_{2}\right\}$ separates two cliques having weight $2 k$ also.

Note that our results only apply in the Euclidean plane; they do not generalize. In particular, Capoyleas et al. 4] give an "unseparable" instance in $\mathbb{R}^{3}$. Our result in the weighted case also is restricted to the plane; the concept of co-bipartite neighborhood edge elimination ordering (CNEEO) does not generalize to $\mathbb{R}^{3}$.

\section{Acknowledgments}

We thank Sriram Pemmaraju, Lorna Stewart, and Zoya Svitkina for helpful discussions. Our thanks to an anonymous source for pointing out the result of Capoyleas et al. [4].

\section{References}

[1] J. Aspnes, D. K. Goldenberg, and Y. R. Yang. On the computational complexity of sensor network localization. In ALGOSENSORS '04: First International Workshop on Algorithmic Aspects of Wireless Sensor Networks, pages 32-44, Turku, Finland, 2004. Springer-Verlag.

[2] B. Balasundaram and S. Butenko. Optimization problems in unit-disk graphs. In Christodoulos A. Floudas and Panos M. Pardalos, editors, Encyclopedia of Optimization, pages 2832-2844. Springer, 2009. 
[3] L. Becchetti, P. Korteweg, A. Marchetti-Spaccamela, M. Skutella, L. Stougie, and A. Vitaletti. Latency constrained aggregation in sensor networks. In Yossi Azar and Thomas Erlebach, editors, ESA, volume 4168 of Lecture Notes in Computer Science, pages 88-99. Springer, 2006.

[4] V. Capoyleas, G. Rote, and G. J. Woeginger. Geometric clusterings. J. Algorithms, 12(2):341$356,1991$.

[5] M. R. Cerioli, L. Faria, T. O. Ferreira, C. A. J. Martinhon, F. Protti, and B. Reed. Partition into cliques for cubic graphs: Planar case, complexity and approximation. Discrete Applied Mathematics, 156(12):2270-2278, 2008.

[6] M.R. Cerioli, L. Faria, T.O. Ferreira, and F. Protti. On minimum clique partition and maximum independent set on unit disk graphs and penny graphs: complexity and approximation. Electronic Notes in Discrete Mathematics, 18:73-79, 2004.

[7] G. Finke, V. Jost, M. Queyranne, and A. Sebö. Batch processing with interval graph compatibilities between tasks. Discrete Applied Mathematics, 156(5):556 - 568, 2008.

[8] D. Gijswijt, V. Jost, and M. Queyranne. Clique partitioning of interval graphs with submodular costs on the cliques. Operations Research, 41(3):275-287, jul 2007.

[9] F. Kuhn, T. Moscibroda, T. Nieberg, and R. Wattenhofer. Fast deterministic distributed maximal independent set computationon growth-bounded graphs. In Pierre Fraigniaud, editor, DISC, volume 3724 of Lecture Notes in Computer Science, pages 273-287. Springer, 2005.

[10] F. Kuhn, T. Nieberg, T. Moscibroda, and R. Wattenhofer. Local approximation schemes for ad hoc and sensor networks. In DIALM-POMC, pages 97-103, 2005.

[11] F. Kuhn and R. Wattenhofer. On the complexity of distributed graph coloring. In Eric Ruppert and Dahlia Malkhi, editors, PODC, pages 7-15. ACM, 2006.

[12] K. M. Lillis, S. V. Pemmaraju, and I. A. Pirwani. Topology control and geographic routing in realistic wireless networks. In Evangelos Kranakis and Jaroslav Opatrny, editors, ADHOCNOW, volume 4686 of Lecture Notes in Computer Science, pages 15-31. Springer, 2007.

[13] T. Nieberg, J. Hurink, and W. Kern. Approximation schemes for wireless networks. ACM Transactions on Algorithms, 4(4):1-17, 2008.

[14] S. Pandit and S. Pemmaraju. Finding facilities fast. In ICDCN, 2009.

[15] S. Pandit, S. Pemmaraju, and K. Varadarajan. Approximation algorithms for domatic partition. In RANDOM-APPROX to appear, 2009.

[16] D. Peleg. Distributed computing: a locality-sensitive approach. Society for Industrial and Applied Mathematics, Philadelphia, PA, USA, 2000.

[17] S. V. Pemmaraju and I. A. Pirwani. Energy conservation via domatic partitions. In Sergio Palazzo, Marco Conti, and Raghupathy Sivakumar, editors, MobiHoc, pages 143-154. ACM, 2006. 
[18] S. V. Pemmaraju and I. A. Pirwani. Good quality virtual realization of unit ball graphs. In Lars Arge, Michael Hoffmann, and Emo Welzl, editors, ESA, volume 4698 of Lecture Notes in Computer Science, pages 311-322. Springer, 2007.

[19] I. A. Pirwani and M. R. Salavatipour. A ptas for minimum clique partition in unit disk graphs. CoRR, abs/0904.2203, 2009.

[20] V. Raghavan and J. Spinrad. Robust algorithms for restricted domains. J. Algorithms, 48(1):160-172, 2003.

[21] J. Schneider and R. Wattenhofer. A log-star distributed maximal independent set algorithm for growth-bounded graphs. In Rida A. Bazzi and Boaz Patt-Shamir, editors, PODC, pages 35-44. ACM, 2008.

[22] D. Zuckerman. Linear degree extractors and the inapproximability of max clique and chromatic number. Theory of Computing, 3(1):103-128, 2007. 